\title{
LAMINAR FLOW PAST AN OSCILLATING CIRCULAR CYLINDER IN CROSS FLOW
}

Pham Anh-Hung

Department of Naval Architecture and Ocean Engineering, Pusan National University, San 30, Jangjeon-Dong, Gumjeong-Gu, Busan, 609-735, Korea

Lee Chang-Yeol

Department of Naval Architecture and Ocean Engineering, Pusan National University, San 30, Jangjeon-Dong, Gumjeong-Gu, Busan, 609-735, Korea

Seo Jang-Hoon Department of Naval Architecture and Ocean Engineering, Pusan National University, San 30, Jangjeon-Dong, Gumjeong-Gu, Busan, 609-735, Korea

Chun Ho-Hwan

Department of Naval Architecture and Ocean Engineering, Pusan National University, San 30, Jangjeon-Dong, Gumjeong-Gu, Busan, 609-735, Korea

Kim Hee-Jung Department of Naval Architecture and Ocean Engineering, Pusan National University, San 30, Jangjeon-Dong, Gumjeong-Gu, Busan, 609-735, Korea

See next page for additional authors

Follow this and additional works at: https://jmstt.ntou.edu.tw/journal

Part of the Ocean Engineering Commons

\section{Recommended Citation}

Anh-Hung, Pham; Chang-Yeol, Lee; Jang-Hoon, Seo; Ho-Hwan, Chun; Hee-Jung, Kim; Yoon, Hyun-Sik; Park, Dong-Woo; and Park, II-Ryong (2010) "LAMINAR FLOW PAST AN OSCILLATING CIRCULAR CYLINDER IN CROSS FLOW," Journal of Marine Science and Technology. Vol. 18: Iss. 3, Article 5.

DOI: 10.51400/2709-6998.1881

Available at: https://jmstt.ntou.edu.tw/journal/vol18/iss3/5

This Research Article is brought to you for free and open access by Journal of Marine Science and Technology. It has been accepted for inclusion in Journal of Marine Science and Technology by an authorized editor of Journal of Marine Science and Technology. 


\section{LAMINAR FLOW PAST AN OSCILLATING CIRCULAR CYLINDER IN CROSS FLOW}

\section{Acknowledgements}

This work was supported by Advanced Ship Engineering Research Center (ASERC) of Pusan National University through Korea Science and Engineering Foundation.

\section{Authors}

Pham Anh-Hung, Lee Chang-Yeol, Seo Jang-Hoon, Chun Ho-Hwan, Kim Hee-Jung, Hyun-Sik Yoon, DongWoo Park, and II-Ryong Park 


\title{
LAMINAR FLOW PAST AN OSCILLATING CIRCULAR CYLINDER IN CROSS FLOW
}

\author{
Anh-Hung Pham*, Chang-Yeol Lee*, Jang-Hoon Seo*, Ho-Hwan Chun*, Hee-Jung Kim*, \\ Hyun-Sik Yoon**, Jeong-Hu Kim***, Dong-Woo Park***, and Il-Ryong Park****
}

Key words: oscillating cylinder, vortex shedding, wake structure, drag, lift.

\begin{abstract}
The present study numerically investigates the twodimensional laminar flow past a circular cylinder forced to oscillate transverse to the free-stream. The numerical simulations are performed at a various range of cylinder oscillation frequency ranged between 0.8 and 1.2 of the natural vortex shedding frequency, and the oscillation amplitude extended up to $50 \%$ of the cylinder diameter at one Reynolds number of 185 showing the typical two-dimensional vortex shedding. The immersed boundary method is used to handle the oscillating cylinder in a rectangular grid system using the finite volume method. The primary vortex shedding frequency has the same value with the exciting frequency. When the exciting frequency exceeds the natural vortex shedding frequency, the secondary vortex shedding frequency appeared with the value less than the natural shedding frequency. The time sequence of the wake structures near the cylinder at the extreme upper position reveals that a single vortex or a pair of vortices appears according to the time. A pair of vortices is composed of two saddle points and two centers of vortices, showing the occurrence of vortex switching phenomenon. The quantitative information about the flow variables such as the distribution of wall vorticity on the cylinder surface, drag and lift coefficients is highlighted.
\end{abstract}

\section{INTRODUCTION}

There are extensive literatures on the flow over a cylinder

Paper submitted 04/23/09; revised 06/10/09; accepted 06/11/09. Author for correspondence: Hyun-Sik Yoon (e-mail: lesmodel@pusan.ac.kr).

*Department of Naval Architecture and Ocean Engineering, Pusan National University, San 30, Jangjeon-Dong, Gumjeong-Gu, Busan, 609-735, Korea.

**Advanced Ship Engineering Research Center, Pusan National University, San 30, Jangjeon-Dong, Gumjeong-Gu, Busan, 609-735, Korea.

***Hyundai Heavy Industries, 1, Jeonha-Dong, Dong-Gu, Ulsan, 682-792, Korea.

****Maritime and Ocean Engineering Research Institute, KORDI, 171 JangDong, Yuseong-Gu, Daejeon, 305-343, Korea. as it is an important problem for academic and engineering purposes. Building on our understanding of a single stationary cylinder, many researchers have focused on multiple stationary or oscillating cylinders. The tube bundle for heat exchangers is an example of multiple cylinders and some of them experience flow-induced vibrations. Also, oscillating cylinders in cross or stationary flow are frequently observed in offshore structures and power cables that are accompanied with fluid-structure interactions.

Flow past a single oscillating cylinder has been researched by plenty of previous investigators. Toebes [11], Ongoren \& Rockwell [10], Williamson \& Roshko [12] and Gu et al. [4] studied experimentally, and Meneghini \& Bearman [9], Lu \& Dalton [8], Blackburn \& Henderson [3], Anagnostopoulos [1, 2] and Guilmineau \& Queutey [5] investigated numerically. Consequently, these researches have greatly contributed to providing a better understanding of the flow characteristics governed by the amplitude and frequency of the oscillation.

Ongoren and Rockwell [10] experimentally showed that the switch of vortex formation position occurs according to the oscillation frequency. Namely, for frequencies of oscillation above the natural shedding frequency, the vortex formed on one side of the cylinder was shed on the opposite side when the cylinder reaches the maximum amplitude of oscillation. As the oscillation frequency is smaller than the natural shedding frequency, the vortex is shed when the cylinder reaches the maximum amplitude on the same side.

Williamson and Roshko [12] experimentally analyzed the wake patterns behind an oscillating cylinder at low Reynolds number. They described vortices' formation and shedding in detail along with the moving cylinder. In the fundamental lock-in region oscillating cylinder sheds four vortices each cycle. They classified the wake patterns with sign ' $\mathrm{P}$ ' for a vortex pair and ' $\mathrm{S}$ ' for a single vortex. Below a critical trajectory wave length, cylinder creates $2 \mathrm{~S}$ mode of the Karman street type. If the wave length is increased over a critical value, the cylinder sheds two like-signed vortices pairs (2P mode).

$\mathrm{Gu}$ et al. [4] experimentally investigated the flow over an oscillating cylinder for two Reynolds numbers of 185 and 5,000 . The frequency ratios, which are defined as an oscillating frequency over a natural vortex shedding frequency of the fixed cylinder, range from 0.8 to 1.2 . As the frequency 
ratio increases, the vortex formation length decreases and if the frequency ratio goes over 1.12, the switch of vortex formation position occurs and persists up to frequency ratio of 1.2 .

Lu \& Dalton [8] and Guilmineau \& Queutey [5] numerically simulated the flow over an oscillating cylinder at $\mathrm{Re}=$ 185 to reproduce the experimental results of $\mathrm{Gu}$ et al. [4]. They also observed the vortex switching phenomenon and explained it with detailed numerical data such as the distribution of vorticity along the cylinder and the drag and lift coefficients.

Anagnostopoulos [1, 2] conducted a numerical study of flow past a cylinder forced to oscillate transversely to the incident stream at $\mathrm{Re}=106$. Their numerical results also confirmed the switch in timing of vortex shedding observed in experimental studies. When the switch in timing of vortex shedding occurs, the flow is aperiodic at subsequent cycles. This aperiodicity is initiated by an instability in the near-wake, due to the coexistence of two saddle points in the streamline pattern, in the instant when the cylinder displacement becomes maximum.

As reviewed above, each effect of the amplitude and frequency of the oscillation on the flow has been quite well established by numerous researches, resulting in greatly improving the understanding of the flow characteristics.

Thus, this study aims at providing the detail quantitative information about the flow variables such as the drag and lift coefficients, shedding frequency, and wall vorticity distributions on the cylinder in the wide range of the amplitude and frequency of the oscillation.

\section{NUMERICAL APPROACH}

The immersed boundary method is used to simulate two-dimensional flow past an oscillating circular cylinder. Therefore, the governing equations describing unsteady incompressible viscous flow field in the present study are the momentum and continuity equations:

$$
\begin{gathered}
\frac{\partial u_{i}}{\partial t}+\frac{\partial u_{i} u_{j}}{\partial x_{j}}=-\frac{\partial p}{\partial x_{i}}+\frac{1}{\operatorname{Re}} \frac{\partial^{2} u_{i}}{\partial x_{j}^{2}}+f_{i} \\
\frac{\partial u_{i}}{\partial x_{i}}-q=0
\end{gathered}
$$

where $x_{i}$ are Cartesian coordinates, $u_{i}$ are the corresponding velocity components, $t$ is the time, and $p$ is the pressure. The momentum forcing $f_{i}$ and mass source/sink $q$ are applied on the body surface or inside the body to satisfy the no-slip condition and mass conservation in the cell containing the immersed boundary.

All the variables are nondimensionalized by the cylinder diameter $D$ and free stream velocity $U_{\infty}$. The above nondimensionalization results in a dimensionless parameter of

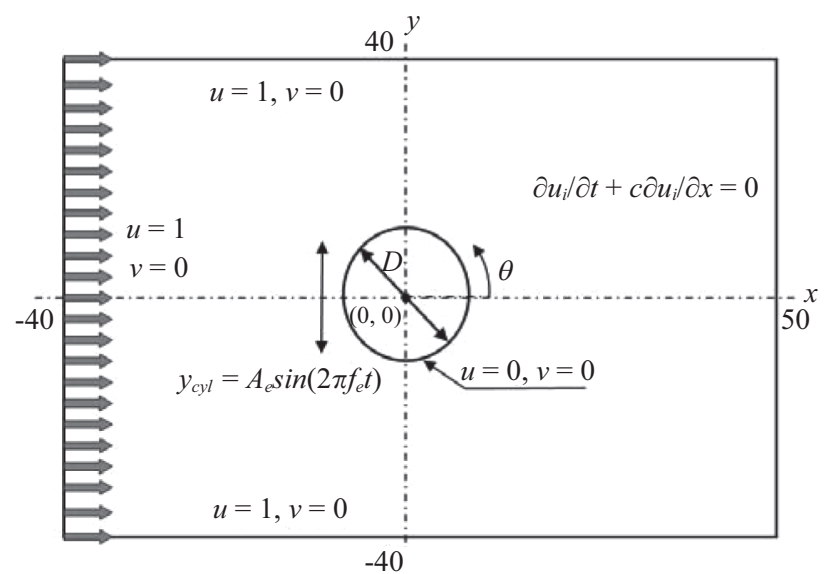

(a)

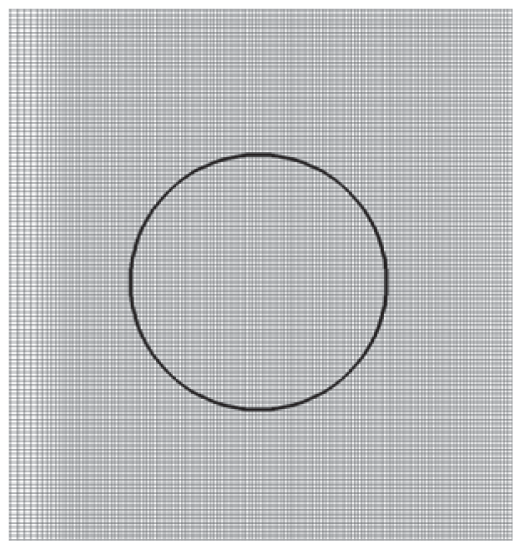

(b)

Fig. 1. (a) Schematic diagram of computational domain and coordinate system along with boundary conditions, and (b) mesh near a cylinder.

$\operatorname{Re}=U_{\infty} D / v$. The Reynolds number of $\operatorname{Re}=185$ is considered in this study.

A finite volume method is used in the present study where the second-order two-step fractional step method is employed for time advancement. The scheme was used previously by Kim and Moin [7] and Zang et al. [15]. The nonlinear terms are treated explicitly using second-order Adams-Bashforth scheme and diffusion terms are treated implicitly using Crank-Nicolson scheme. The central difference scheme with the second-order accuracy based on the finite volume method is used for the spatial discretization. Additionally, a second-order linear or bilinear interpolation scheme is applied to satisfy the no-slip condition on the immersed boundary. Further details of the immersed-boundary method are given in Kim et al. [6].

Figure 1 shows the computational domain, coordinates system and grid distribution near the cylinder. The size of computational domain is $-40 \leq x \leq 50$ and $-40 \leq x \leq 40$ for the streamwise and transverse directions, respectively. At the inflow and far-field boundaries, the Dirichlet boundary conditions, $u=1$ and $v=0$, are enforced. The convective bound- 
ary condition, $\partial u_{i} / \partial t+c \partial u_{i} / \partial x=0$, is applied at the outflow boundary, where $c$ is the space-averaged exit velocity.

As shown in Fig. 1, the trajectory of cylinder center along $y$ direction is imposed as $y_{c y l}(t)=-A_{e} \sin \left(2 \pi f_{e} t\right)$ based on Guilmineau \& Queutey [5]'s study, where $A_{e}$ is oscillating amplitude and $f_{e}$ is the exciting frequency. The oscillation amplitude normalized by the cylinder diameter has changed in four cases having the values $0.2,0.3,0.4$ and 0.5 . The frequency ratio, $f_{r}=f_{e} / f_{o}$, where $f_{o}$ is the natural shedding frequency of a fixed cylinder at $\mathrm{Re}=185$, ranges from 0.8 to 1.2 .

The number of total grid points used is $483(x) \times 451(y)$. The grids are nonuniformly distributed near the cylinder, especially the wake region to accurately capture the separating shear layers around the cylinder and in order to account for the high gradients near the surfaces. $80 \times 80$ grid points are uniformly distributed inside the cylinder while a hyperbolic tangent distribution is in the outer regions. Figure 1(b) shows the typical grid distribution near the cylinder. In order to consider the variation of the amplitude of oscillation, the number of grid points used in the $y$ direction is tuned to maintain the dense resolution near the cylinder. Consequently, grid independence of the solution has been confirmed with additional simulations on the finer grids $(683(x) \times 621(y))$ changing the body forces by less than $0.1 \%$. The condition of $C F L<0.15$ is chosen to determine the nondimensional time step used in the present calculations.

The computations were advanced in time until it was observed that the drag and lift coefficients have reached a statistically stationary state. The effect of calculation time on the fully developed state has been examined using the different initial conditions. Although the simulations start with the different initial conditions, the fully developed steady or periodic flows are reached after about 100 time units. Every computation is extended to $t \geq 200$ to minimize the effect of calculation time on the final flow state.

The present numerical method has been adopted to simulate flows past a rotating circular cylinder and around two stationary circular cylinders of the same diameter in a side-byside arrangement by Yoon et al. [14] \& [13]. Consequently, the results obtained by the present method gave a good agreement with those of previous researches (Yoon et al. [14] \& [13]), which confirms the reliability of the present numerical method. Also, the present results for the oscillating cylinder are in good agreement with those of previous studies, which will be appeared in the part of results and discussion.

\section{RESULTS ANS DISCUSSION}

The time histories of drag and lift coefficients $\left(C_{D}\right.$ and $\left.C_{L}\right)$ at the different oscillating amplitudes for $f_{r}$ of 0.8 and 1 are plotted in the left and right column of Fig. 2, respectively. Even if $A_{e} / D$ changes in the range from 0.2 to 0.5 , the dependence of the time histories of $C_{D}$ and $C_{L}$ on the $f_{r}$ within the limits considered in this study is generally the same as follows.
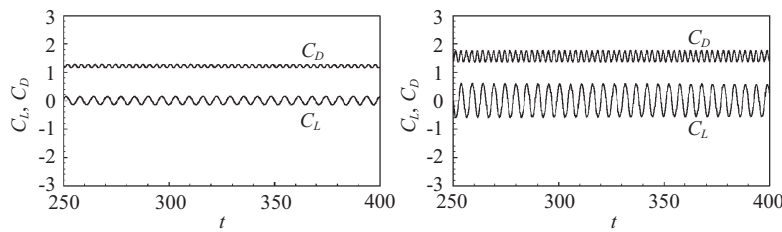

(a)
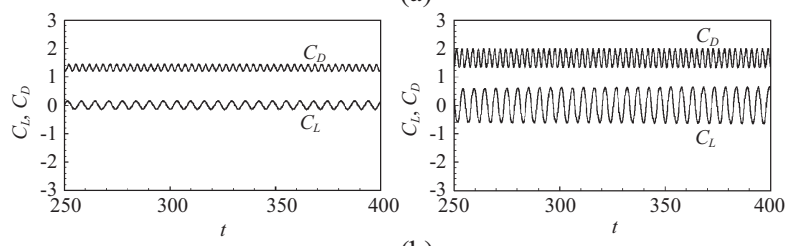

(b)
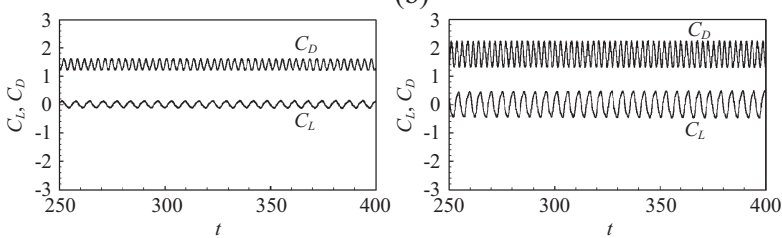

(c)
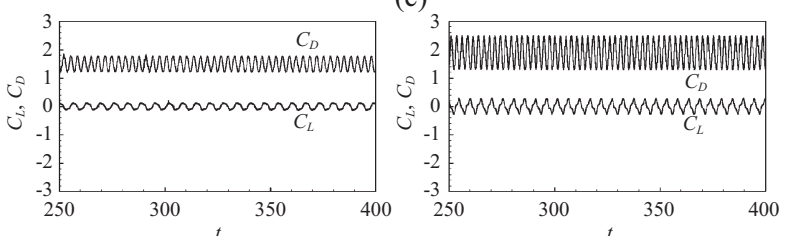

(d)

Fig. 2. Time histories of drag and lift coefficients at $f_{r}=0.8$ (left column) and $f_{r}=1.0$ (right column) for different $A_{e} / D$ s: (a) $A_{e} / D=0.2$, (b) $A_{e} / D=0.3$, (c) $A_{e} / D=0.4$, (d) $A_{e} / D=0.5$.

When $f_{r} \leq 1, C_{D}$ and $C_{L}$ are time-dependent and regularly repeated in a periodic fashion regardless of $A_{e} / D$. However, the amplitude of the time histories of $C_{D}$ and $C_{L}$ has the dependence on $f_{r}$ and also $A_{e} / D$ at the fixed $f_{r}$.

To clarify the frequency of the vortex shedding, the power spectra of the time histories of $C_{L}$ at different $A_{e} / D$ s for different $f_{r}$ s are displayed in Fig. 4 . In the cases of $f_{r}=0.8$ and 1 , the power spectra of the time histories of $C_{L}$ show clearly that a single frequency is the same as that of the cylinder oscillating frequency as shown in Figs. 4(a) and 4(b), respectively.

The time histories of $C_{D}$ and $C_{L}$ at the different oscillating amplitudes for $f_{r} \mathrm{~s}$ of 1.1 and 1.2 are plotted in the left and right columns of Fig. 3, respectively. Here, only the cases of $f_{r}=1.1$ and 1.2 among $f_{r}>1$ are presented to show clearly the effect of exciting frequency on the evolution of the drag and lift forces at the different $A_{e} / D \mathrm{~s}$. When $f_{r}>1$, the time traces of $C_{D}$ and $C_{L}$ become modulated but shows beats for all oscillating amplitudes.

The power spectra of $C_{L}$ at different $A_{e} / D$ s for $f_{r}=1.1$ and 1.2 are shown in Figs. 4(c) and 4(d), respectively. These power spectra show the dominant nondimensional primary frequency of 1 with harmonics and indicate a lot of vortices activities with the presence of low frequency oscillations in the $C_{L}$ signal as shown in Fig. 3 . 

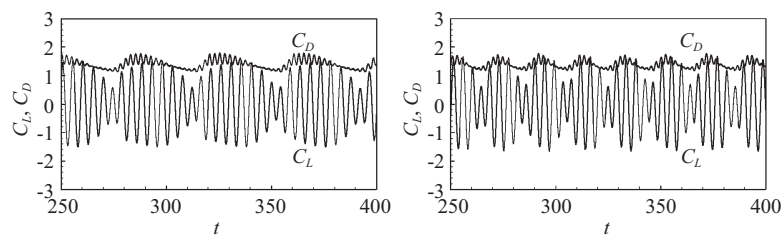

(a)
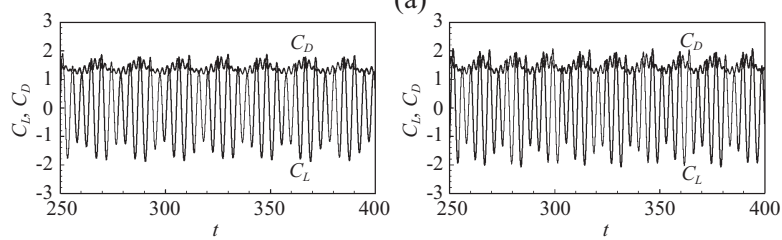

(b)
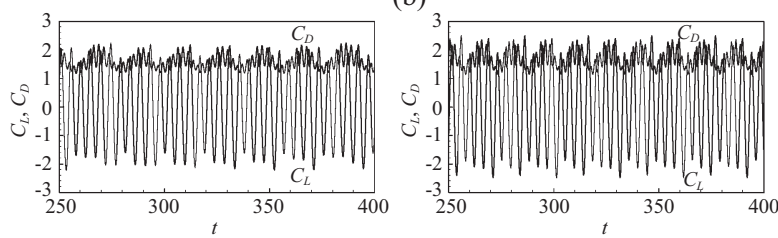

(c)
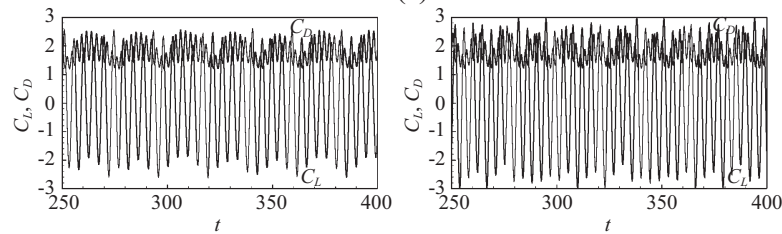

(d)

Fig. 3. Time histories of drag and lift coefficients at $f_{r}=1.1$ (left column) and $f_{r}=1.2$ (right column) for different $A_{e} / D$ s: (a) $A_{e} / D=0.2$, (b) $A_{e} / D=0.3$, (c) $A_{e} / D=0.4$, (d) $A_{e} / D=0.5$.

The dependence of the time traces of $C_{D}$ and $C_{L}$ on $A_{e} / D$ and $f_{r}$ obtained from the present study is consistent with the findings of previous studies $([1,2,5,8])$ even with the different Reynolds numbers.

In order to clarify the dependence of the shedding frequency $\left(f_{s}\right)$ defined by the frequency of $C_{L}$ shown in Fig. 4 on the $A_{e} / D$ and $f_{r}$, the normalized $f_{s}$ by the natural shedding frequency is plotted together as a function of the $f_{r}$ for different $A_{e} / D \mathrm{~s}$ in Fig. 5, where $f_{s, P}$ and $f_{s, S}$ are the primary and secondary frequencies of $f_{s}$, respectively. For all the $A_{e} / D \mathrm{~s}$, the primary frequency has the same value with the exciting frequency $\left(f_{e}\right)$. In other word, $f_{s, P}$ is the linear relation with $f_{r}$, regardless of $A_{e} / D$. When $f_{r}$ exceeds 1 , the secondary frequency for all the $A_{e} / D$ s appears with the value less than the natural shedding frequency. For $A_{e} / D=0.2$, in the range of $1 \leq f_{r} \leq 1.2, f_{s, S}$ has almost the same value with about $5 \%$ less than that of $f_{o}$. In the cases of $A_{e} / D=0.3,0.4$ and 0.5 , the value of $f_{s, S}$ rapidly decreases and distributes in the narrow range of $85 \% \sim 87 \%$ of $f_{o}$.

Figure 6 shows the instantaneous vorticity contours at different $A_{e} / D$ s for $f_{r}=0.8$ and 1.0 when the oscillating cylinder is at the uppermost position. When the frequency ratio is fixed, the formation of vortices in the wake region for the different $A_{e} / D \mathrm{~s}$ is generally identical. Also the spacing of vortices with different signs almost keeps in both the longitu-
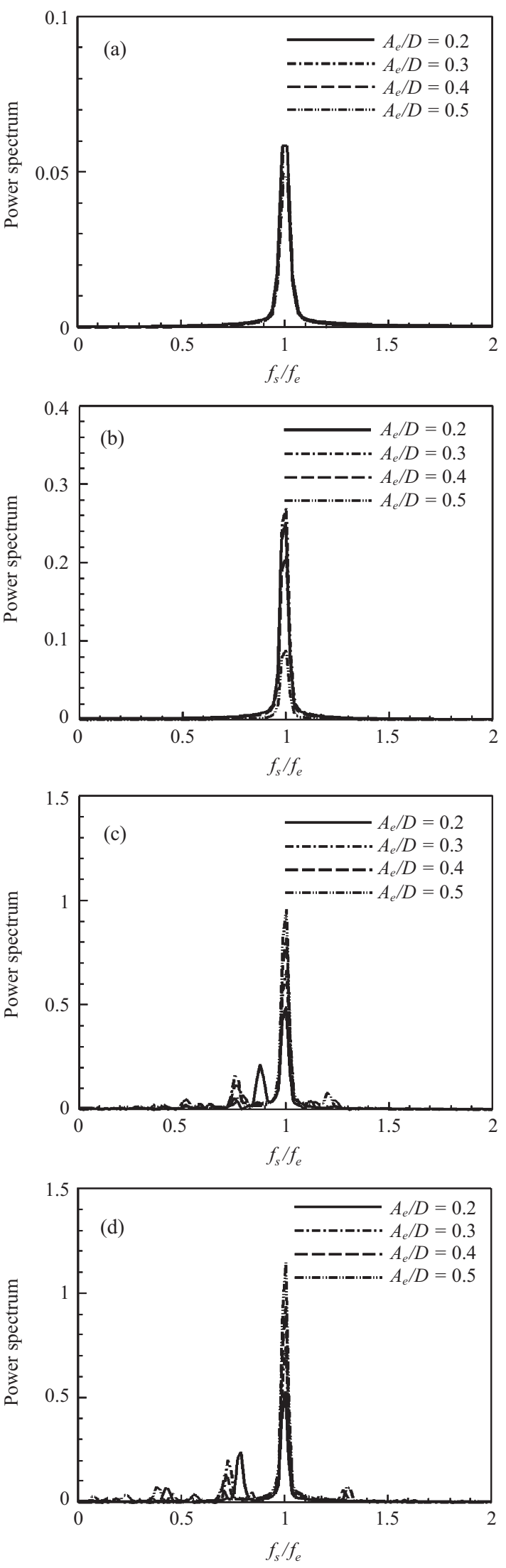

Fig. 4. Power spectra as a function of the frequency ratio $\left(f_{s} / f_{e}\right)$ for different $A_{e} / D$ s: (a) $f_{r}=0.8$, (b) $f_{r}=1.0$, (c) $f_{r}=1.1$, (d) $f_{r}=1.2$. 


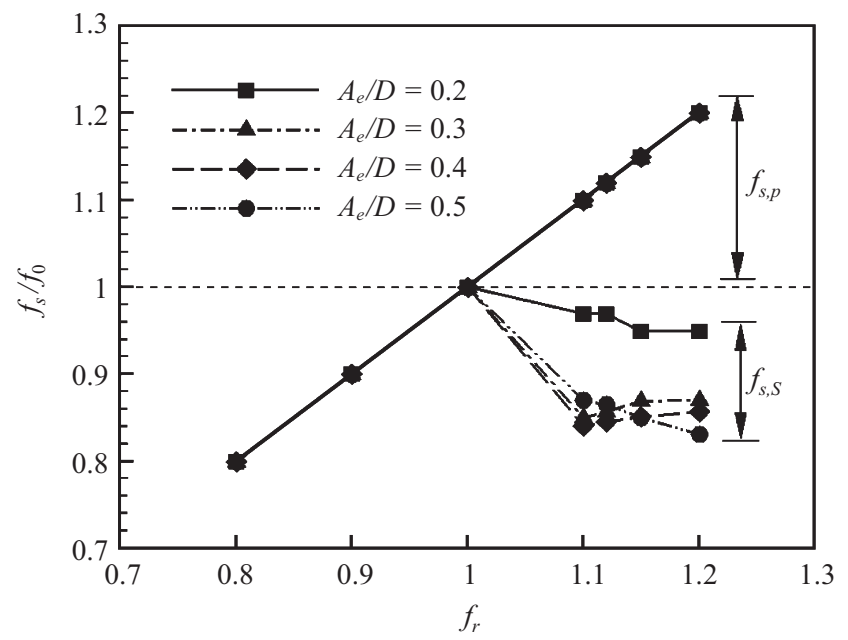

Fig. 5. Normalized vortex shedding frequency $f_{s}$ by the natural shedding frequency $f_{o}$ as a function of as a function of the $f_{r}$ for different $A_{e} / D \mathrm{~s}$, where $f_{s, P}$ and $f_{s, S}$ are the primary and secondary frequencies of $f_{S}$, respectively.

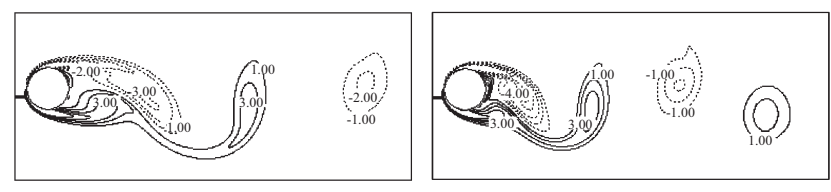

(a)

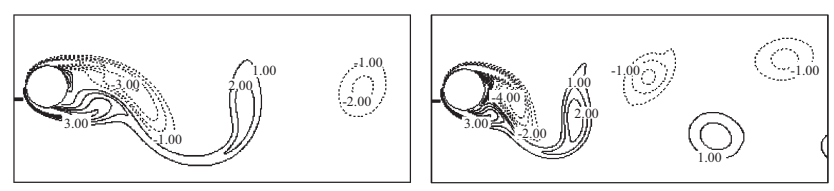

(b)
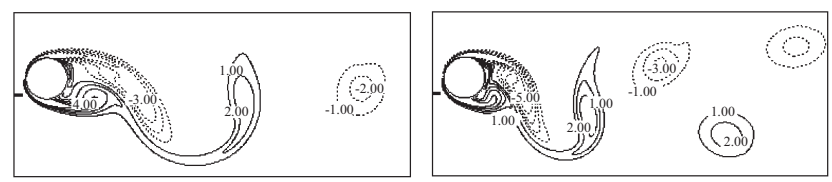

(c)
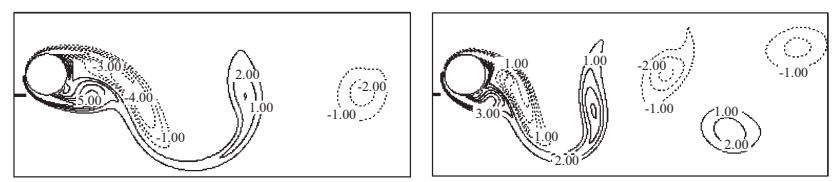

(d)

Fig. 6. Instantaneous vorticity contours at the extreme upper position for $f_{r}=0.8$ (left column) and $f_{r}=1.0$ (right column) for different $A_{e} / D$ s: (a) $A_{e} / D=0.2$, (b) $A_{e} / D=0.3$, (c) $A_{e} / D=0.4$, (d) $A_{e} / D=0.5$.

dinal and lateral directions regardless of the variation of $A_{e} / D$. However, as $A_{e} / D$ increases, the vortices more incline and elongate along the lateral direction.

When $A_{e} / D$ is fixed, the longitudinal distance of vortices becomes shorter and consequently the vorticities are much concentrated near the rear of cylinder with increasing $f_{r}$. Contrary to the longitudinal spacing of vortices, the lateral

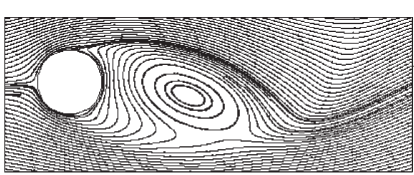

(a)

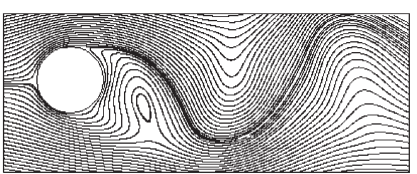

(b)

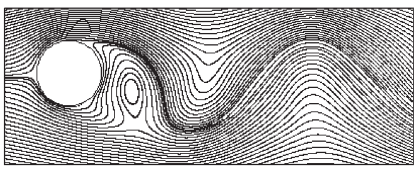

(c)

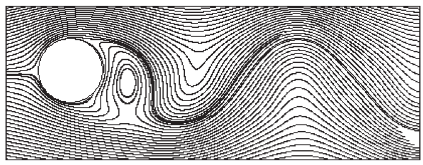

(d)

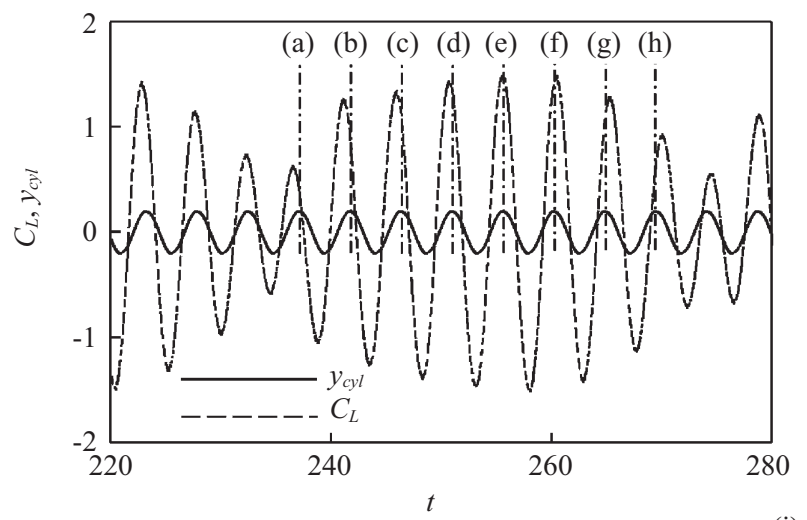

(i)

Fig. 7. Time sequence of eight streamlines at the extreme upper position during one period of lift coefficient for $f_{r}=1.1$ at $A_{e} / D=0.2$. Here, the corresponding instant is marked in the plot of time history of lift coefficient and the trajectory of cylinder center $\left(C_{L}\right.$ and $\left.y_{c y l}\right)$.

spacing of vortices with different signs becomes wider with increasing $f_{r}$.

Representatively, for $f_{r}=1.1$ at $A_{e} / D=0.2$, the time sequence of eight instantaneous streamlines at the upper position during eight oscillating period of cylinder are plotted in Fig. 7. As expected from Fig. 3 showing that the time histories of $C_{D}$ and $C_{L}$ lost the regular periodicity but showed beats for all oscillating amplitudes, it can be clearly identified that the vortex formation in the wake depends on the time even though the cylinder locates at the same position.

When the cylinder locates at the extreme upper position during the eight oscillating period of cylinder, the wake structures near the rear of cylinder are composed of a single vortex 


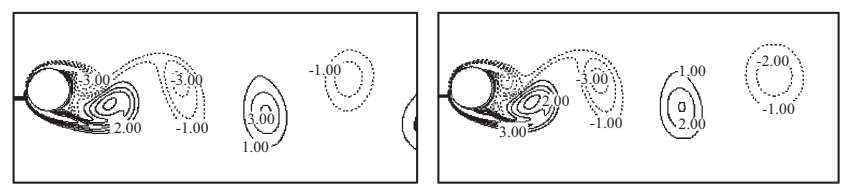

(a)
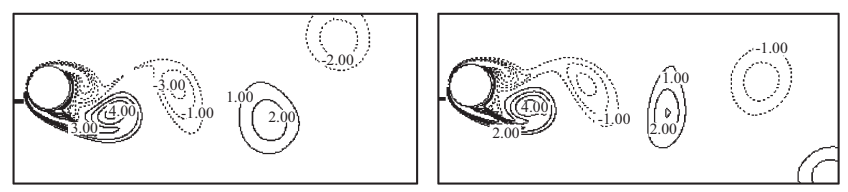

(b)

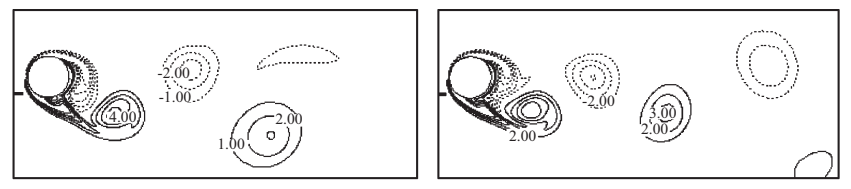

(c)
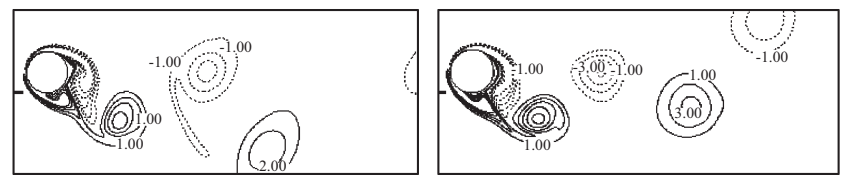

(d)

Fig. 8. Instantaneous vorticity contours at the extreme upper position at $f_{r}=1.1$ (left column) and $f_{r}=1.2$ (right column) for different $A_{e} / D$ s: (a) $A_{e} / D=0.2$, (b) $A_{e} / D=0.3$, (c) $A_{e} / D=0.4$, (d) $A_{e} / D=0.5$.

or a pair of vortices. Especially, Figs. 7(f) and 7(g) show clearly two saddle points and two centers of vortices, which is the one of the criterions for the occurrence of vortex switching phenomenon $([5,8])$. These coexistence of two saddle points and two centers of vortices result in the instability in the wake or aperiodicity.

Figure 8 shows the instantaneous vorticity contours for $f_{r}=1.1$ and 1.2 at different $A_{e} / D$ s when the oscillating cylinder is at the extreme upper position. When $f_{r}$ exceeds the natural shedding frequency of 1 , the vertical shear layers induced by the cylinder oscillating reduce the strength of upper and lower vorticities and limit the vorticity evolution in the free stream direction. Consequently, the upper vorticity becomes smaller in the longitudinal direction. The longitudinal spacing between the vorticities in the wake region decreases. Successively, near the wake, the series of vorticities with different signs more concentrate than the cases of lower $f_{r}$.

As $A_{e} / D$ increases at the fixed $f_{r}$, the negative vorticity shed from the upper surface elongates more along the cylinder surface. Although $A_{e} / D$ increases, the longitudinal spacing between the vorticities in the wake region keeps about the same. Also, the upper negative vorticity increases in strength and concentration. The effect of the frequency ratio on this flow pattern is in full agreement with the experimental results by Ongoren \& Rockwell [10] and Gu et al. [4] who showed that an increase in the longitudinal spacing of the vortices in the near-wake is observed with decreasing frequency ratio.

Figure 9 shows the distribution of time-averaged wall vor-

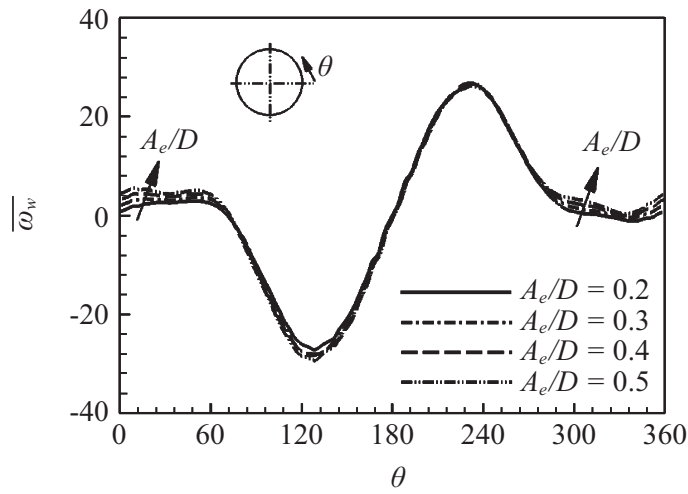

(a)

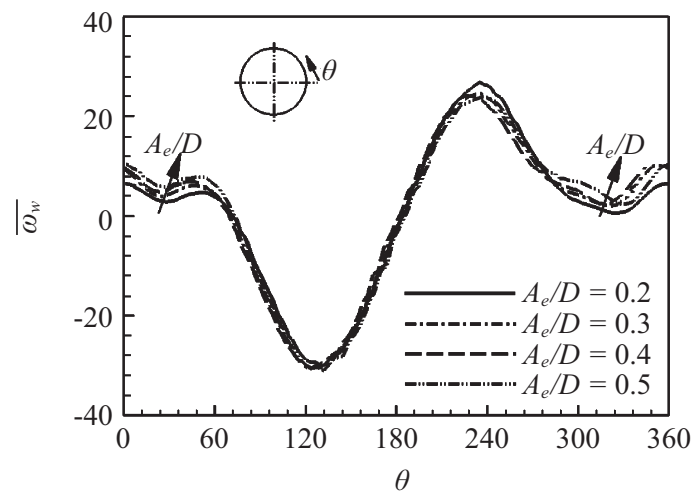

(b)

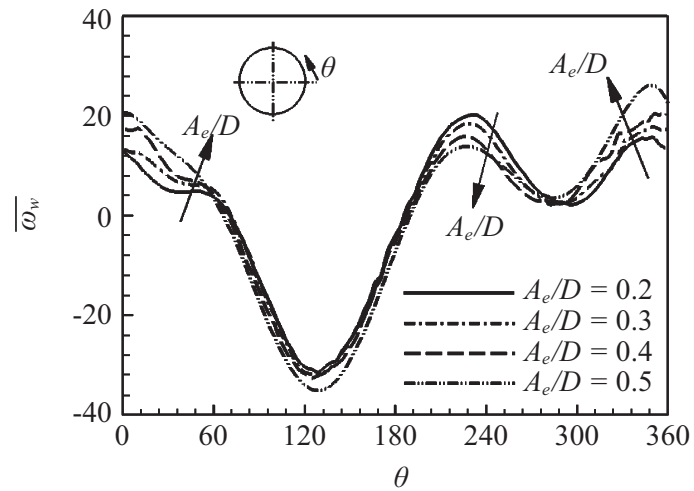

(c)

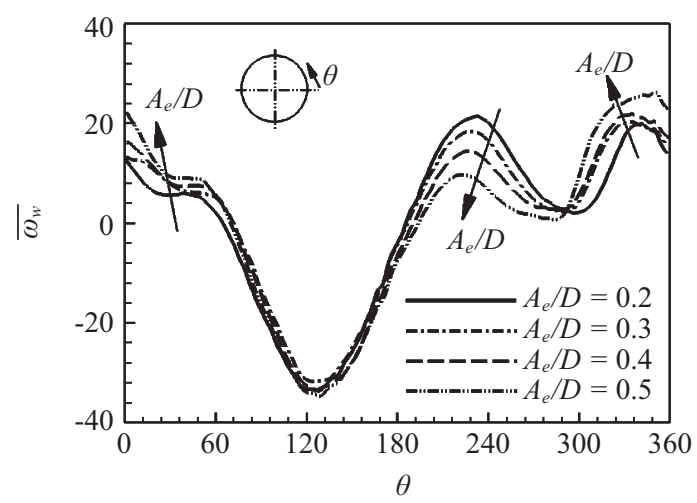

(d)

Fig. 9. Time-averaged wall vorticity along the cylinder surface at the extreme upper position for different $A_{e} / D \mathrm{~s}$ : (a) $f_{r}=0.8$, (b) $f_{r}=1.0$, (c) $f_{r}=1.1$, (d) $f_{r}=1.2$. 
ticity $\left(\overline{\omega_{w}}\right)$ around the cylinder surface for different oscillating amplitude at four different frequency ratios. As $f_{r}$ corresponding to the cylinder oscillatory velocity increases, the base vorticity increases significantly in strength and size, which has been observed in between the cylinder surface and the upper negative vorticity as early shown in Figs. 6 and 8. Especially, for $f_{r}=1.2$, this base vorticity covers the wide range of $0 \leq \theta<60$ and $290<\theta \leq 360$ as shown in Fig. 9(d).

Owing to this base vorticity, the upper vorticity diminishes in the longitudinal evolution or length and strength with increasing $f_{r}$. Also, this upper negative vorticity depends on the oscillatory amplitude, leading to the augment of the magnitude and size with increasing $A_{e} / D$ at the fixed $f_{r}$.

The time-averaged drag coefficient $\left(\overline{C_{D}}\right)$ and root-meansquare (RMS) values of drag and lift coefficients $\left(C_{D r m s}\right.$ and $C_{\text {Lrms }}$ ) as a function of frequency ratio for four different oscillating amplitudes are plotted in Figs. 10(a), 10(b) and 10(c), respectively.

For $A_{e} / D=0.2$, present results of $\overline{C_{D}}, C_{D r m s}$ and $C_{\text {Lrms }}$ agree well with those of Guilmineau \& Queutey [10]. The value of $\overline{C_{D}}$ increases with increasing $f_{r}$ up to 1 where $\overline{C_{D}}$ has a maximum. When $f_{r}$ reaches to $1.1, \overline{C_{D}}$ decreases quickly and further increasing $f_{r}$, it slightly decreases.

In the cases of $A_{e} / D=0.3,0.4$ and 0.5 , the variation of $\overline{C_{D}}$ depending on the frequency ratio is about the same pattern as follows. The increasing behavior of $\overline{C_{D}}$ in the $0.8 \leq f_{r} \leq 1$ is the same pattern with case of $A_{e} / D=0.2$. Also, the peak of $\overline{C_{D}}$ for these $A_{e} / D$ s appears at $f_{r}=f_{o}=1$. When $f_{r}$ reaches to 1.1, $\overline{C_{D}}$ diminishes rapidly. In contrast to $A_{e} / D=0.2$, when $f_{r}$ exceeds 1.1, $\overline{C_{D}}$ increases again as shown in Fig. 10(a).

For $A_{e} / D=0.2, C_{D r m s}$ increases until $f_{r}$ reaches 1.1 where it has a maximum, further increasing $f_{r}$, it slightly decreases. In the cases of $A_{e} / D=0.3,0.4$ and 0.5 , the dependence of $C_{D r m s}$ on $f_{r}$ is the same with the case of $\overline{C_{D}}$, which can be identified by comparing between Fig. 10(a) and Fig. 10(b).

The $C_{\text {Lrms }}$ shows the different behavior along the $f_{r}$ with that of $\overline{C_{D}}$ and $C_{D r m s}$, which can be proved by comparing between Figs. 10(a-b) and Fig. 10(c). For $A_{e} / D=0.2, C_{\text {Lrms }}$ increases with increasing $f_{r}$. When $f_{r}$ reaches $1.1, C_{L r m s}$ rapidly increases, resulting in the steep slope. As $f_{r}$ increases continuously, $C_{\text {Lrms }}$ slightly augments. When $A_{e} / D$ increases, the dependence of $C_{\text {Lrms }}$ on the range of $f_{r}$ is significant. In the range of $0.8 \leq$ $f_{r} \leq 1$, as $A_{e} / D$ increases, $C_{\text {Lrms }}$ profile changes form the linear to the parabolic pattern. Eventually, for $A_{e} / D=0.5$, the minimum of $C_{\text {Lrms }}$ appears at $f_{r}=0.95$.

In the range of $0.8 \leq f_{r} \leq 1$, the value of $C_{L r m s}$ diminishes with increasing $A_{e} / D$ at fixed $f_{r}$. In contrast, when $f_{r}$ is larger than $1, C_{L r m s}$ increases with increasing $A_{e} / D$ at fixed $f_{r}$.

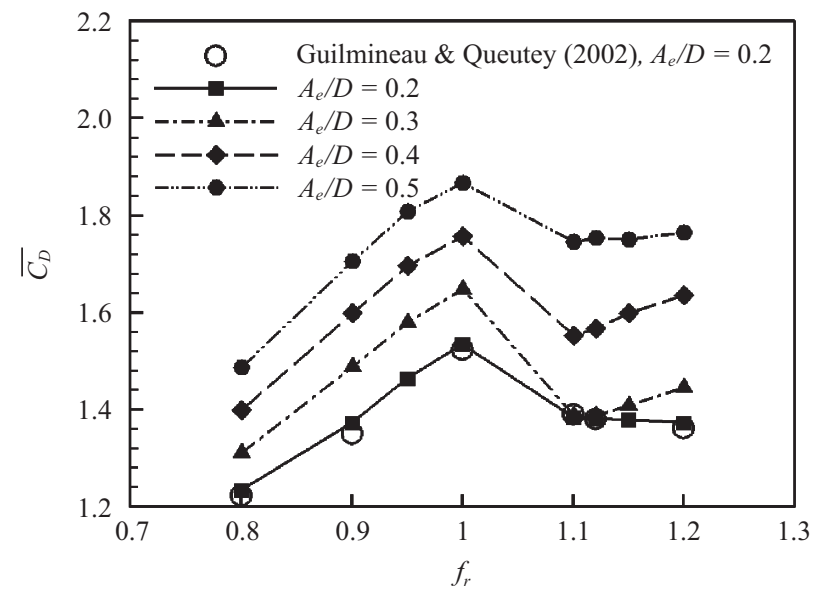

(a)

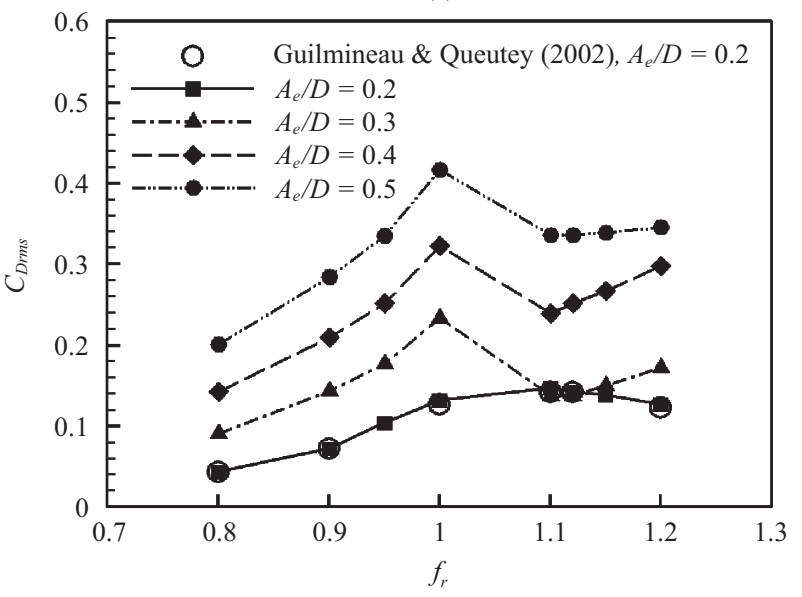

(b)

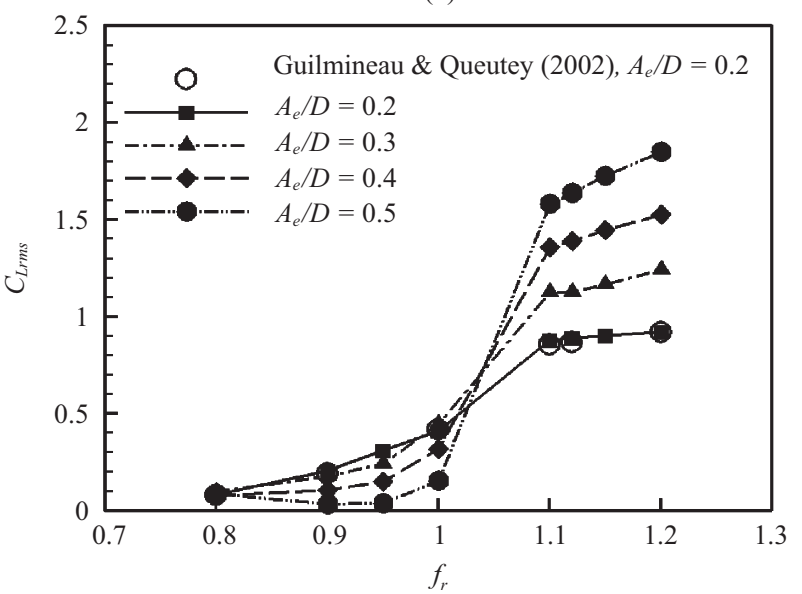

(c)

Fig. 10. Time-averaged drag coefficient $\left(\overline{C_{D}}\right)$ and root-mean-square (RMS) values of drag and lift coefficients $\left(C_{D r m s}, C_{L r m s}\right)$ as a function of frequency ratio for four different oscillating amplitudes: (a) $\overline{C_{D}}$, (b) $C_{D r m s}$, (c) $C_{L r m s}$. For $A_{e} / D=0.2$, present results are compared with those of Guilmineau \& Queutey (2002).

\section{CONCLUSION}

The present study has numerically investigated the char- 
acteristics of two-dimensional laminar flow past a circular cylinder forced to oscillate transverse to the free-stream. In a second-order accurate finite volume method, an immersed boundary method is adopted to handle the cylinder oscillating in the Cartesian coordinates.

The flow has been computed for four different oscillation amplitudes $\left(A_{e} / D\right)$ of $0.2,0.3,0.4$ and 0.5 normalized by the cylinder diameter and the frequency ratio, $f_{r}=f_{e} / f_{o}$, where $f_{e}$ is the excitation frequency of cylinder and $f_{o}$ is the natural shedding frequency of a fixed cylinder at $\mathrm{Re}=185$, ranges from 0.8 to 1.2 .

When $f_{r}$ is less than 1 , the drag and lift coefficients are time-dependent and regularly repeated in a periodic fashion regardless of $A_{e} / D$. However, when $f_{r}$ exceeds 1 , the time histories of $C_{D}$ and $C_{L}$ become modulated and shows beats for all oscillating amplitudes.

The primary vortex shedding frequency has the same value with the exciting frequency, showing the linear relation with $f_{r}$, regardless of $A_{e} / D$. When $f_{r}$ exceeds 1 , the secondary frequency for all the $A_{e} / D$ s appears with the value less than the natural shedding frequency.

When the cylinder is at the extreme upper position, for $f_{r} \leq 1$, the spacing of vortices with different signs almost keeps in both the longitudinal and lateral directions regardless of $A_{e} / D$. For $f_{r}>1$, the vertical shear layers induced by the cylinder oscillating reduce the longitudinal spacing between the vorticities in the wake region.

For $f_{r} \geq 1.1$, the instantaneous wake structures at the extreme upper position revealed a pair of vortices composed of two saddle points and two centers of vortices, showing the occurrence of vortex switching phenomenon and leading to the instability in the wake.

The distribution of time-averaged wall vorticity around the cylinder surface showed that the base vorticity increases significantly in strength and size with increasing $f_{r}$. Owing to this base vorticity, the upper vorticity diminishes in the longitudinal evolution, length and strength with increasing $f_{r}$.

For $A_{e} / D=0.2$, the value of $\overline{C_{D}}$ increases with increasing $f_{r}$ up to 1 where $\overline{C_{D}}$ has a maximum. When $f_{r}$ reaches to 1.1 , $\overline{C_{D}}$ decreases quickly and further increasing $f_{r}$, it slightly decreases. However, $C_{\text {Drms }}$ increases until $f_{r}$ reaches 1.1 where it has a maximum.

In the cases of $A_{e} / D=0.3,0.4$ and $0.5, \overline{C_{D}}$ and $C_{D r m s}$ showed the same dependence on $f_{r}$. Namely, the increasing behavior of $\overline{C_{D}}$ and $C_{\text {Drms }}$ in the $0.8 \leq f_{r} \leq 1$ was observed. When $f_{r}$ reaches to 1.1 , those values diminishes rapidly and further increasing $f_{r}$, they increases again.

The dependence of $C_{L r m s}$ on the range of $f_{r}$ is considerable with increasing $A_{e} / D$. As $A_{e} / D$ increases in the range of $0.8 \leq f_{r} \leq 1$, the profile of $C_{L r m s}$ changes form the linear to the parabolic pattern. Especially, in the case of $A_{e} / D=0.5$, the minimum of $C_{\text {Lrms }}$ appears at $f_{r}=0.95$. In the range of $0.8<f_{r} \leq 1$, the value of $C_{\text {Lrms }}$ diminishes with increasing $A_{e} / D$ at fixed $f_{r}$. In contrast, when $f_{r}$ exceeds $1, C_{L r m s}$ increases with increasing $A_{e} / D$ at fixed $f_{r}$.

\section{ACKNOWLEDGMENTS}

This work was supported by Advanced Ship Engineering Research Center (ASERC) of Pusan National University through Korea Science and Engineering Foundation.

\section{REFERENCES}

1. Anagnostopoulos, P., "Numerical study of the flow past a cylinder excited transversely to the incident stream. Part 1: Lock-in zoned, hydrodynamic forces and wake geometry," Journal of Fluids and Structure, Vol. 14, pp. 819-851 (2000).

2. Anagnostopoulos, P., "Numerical study of the flow past a cylinder excited transversely to the incident stream. Part 2: Timing of vortex shedding, aperiodic phenomena and wake parameters," Journal of Fluids and Structure, Vol. 14, pp. 853-882 (2000).

3. Blackburn, H. M. and Henderson, R. D., "A study of two-dimensional flow past an oscillating cylinder," Journal of Fluid Mechanics, Vol. 385, pp. 255-286 (1999).

4. Gu, W., Chyu, C., and Rockwell, D., "Timing of vortex formation from an oscillating cylinder," Physics of Fluids, Vol. 6, No. 11, pp. 3677-3682 (1994).

5. Guilmineau, E. and Queutey, P., "A numerical simulation of vortex shedding from an oscillating circular cylinder," Journal of Fluids and Structure, Vol. 16, pp. 773-794 (2002).

6. Kim, J., Kim, D., and Choi, H., "An immersed-boundary finite volume method for simulations of flow in complex geometries," Journal of Computational Physics, Vol. 171, pp. 132-150 (2001).

7. Kim, J. and Moin, P., "Application of a fractional step method to incompressible Navier-Stokes equations," Journal of Computational Physics, Vol. 59, pp. 308-323 (1985).

8. Lu, X.-Y. and Dalton, C., "Calculation of the timing of vortex formation from an oscillating cylinder," Journal of Fluids and Structure, Vol. 10, pp. 527-541 (1996).

9. Meneghini, J. R. and Bearman, P. W., "Numerical simulation of high amplitude oscillatory flow about a circular cylinder," Journal of Fluids and Structure, Vol. 9, pp. 435-455 (1995).

10. Ongoren, A. and Rockwell, D., "Flow structure from an oscillating cylinder Part 1. Mechanisms of phase shift and recovery in the near wake," Journal of Fluid Mechanics, Vol. 191, pp. 197-223 (1988).

11. Toebes, G. H., "The unsteady flow and wake near an oscillating cylinder," Journal of Basic Engineering, Vol. 91, pp. 493-505 (1969).

12. Williamson, C. H. K. and Roshko, A., "Vortex formation in the wake of an oscillating cylinder," Journal of Fluids and Structure, Vol. 2, pp. 355-381 (1988).

13. Yoon, H. S., Chun, H. H., Kim, J. H., and Park, I. L., "Flow characteristics of two rotating side-by-side circular cylinder," Computers \& Fluids, Vol. 38, pp. 466-474 (2009).

14. Yoon, H. S., Kim, J. H., Chun, H. H., and Choi , H. J., "Laminar flow past two rotating circular cylinders in a side-by-side arrangement," Physics of Fluids, Vol. 19, pp. 128103-1-4 (2007).

15. Zang, Y., Street, R. L., and Koseff, J. R., “A non-staggered grid, fractional step method for time-dependent incompressible navier-stokes equations in curvilinear coordinates," Journal of Computational Physics, Vol. 114, pp. 18-33 (1994). 